\title{
Tamaño y vegetación de parques urbanos en el cantón Central de San José, Costa Rica
}

\author{
Aarón Fallas Solano \\ Escuela de Ciencias Exactas y Naturales, Universidad Estatal a Distancia (UNED), 2050 San José, Costa Rica; afallas@uned.ac.cr \\ Recibido 06-VII-2017 • Corregido 04-IX-2017 • Aceptado 29-IX-2017
}

\begin{abstract}
Size and vegetation of urban parks in downtown San José, Costa Rica. Worldwide, the natural landscape is being substituted by urban spaces, where green areas are basic for the conservation of many species. I analyzed size and vegetation in 15 urban parks of the Central San José, Costa Rica, in late 2009. Field work and municipal data shows that parks are small ( 1786,82 to $\left.21220,94 \mathrm{~m}^{2}\right)$ and dominated by exotic species ( $87 \%$ of vegetation). Half the parks have an important component of $5 \mathrm{~m}$ tall strata. Carmen district has tree lanes that act as corridors, an example that should be followed by other districts.
\end{abstract}

Key words: Urban parks, biophysical variables, urban fragmentation.

Los paisajes naturales están siendo sustituidos por ambientes urbanos con fines residenciales, comerciales e industriales en las principales capitales del mundo. Este crecimiento urbano provoca cambios en los usos de los suelos, los cuales tienen serias implicaciones para la biodiversidad, creando alteraciones al medio y generando efectos considerables y directos a los ecosistemas naturales (Barillas, 2005). Sin embargo, aún permanecen dentro de las ciudades, parques urbanos o espacios con jardines en la ciudad (Canosa, Sáez, Sanabria \& Zavala, 2003) que permiten mantener fragmentos de cobertura vegetal, ya sean estos de origen natural o establecidos por el humano como parte del paisaje.

Los parques urbanos más comunes pueden ser: parques, áreas verdes, bulevares, jardines, plazas y aceras arboladas, las cuales en cierta medida son respetadas por el ser humano y utilizadas como centros sociales de recreación o esparcimiento. Dichas zonas verdes realizan funciones ecológicas de importancia como mejorar la
RESUMEN: En el mundo, el paisaje natural está siendo sustituido por espacios urbanos, donde las áreas verdes son necesarias para la conservación de muchas especies. Analicé el tamaño y la vegetación en 15 parques urbanos del centro de San José, Costa Rica, a finales del 2009. El trabajo de campo y los datos municipales muestran que los parques son pequeños (1 786,82 a $21220,94 \mathrm{~m}^{2}$ ) y dominados por especies exóticas ( $87 \%$ de vegetación). La mitad de los parques tienen un componente importante de estratos de $5 \mathrm{~m}$ de altura. El distrito del Carmen tiene líneas arbóreas que actúan como corredores, un ejemplo que deberían seguir otros distritos.

Palabras clave: Parques urbanos, variables biofísicas, fragmentación urbana. calidad del aire, el agua y los recursos del suelo al absorber contaminantes, incrementar las áreas de captación y almacenamiento de agua y estabilizar los suelos (Buzo \& Hernández, 2004). Además, actúan como amortiguadores de la temperatura, reducen la contaminación por el ruido y los niveles de dióxido de carbono $\left(\mathrm{CO}_{2}\right)$ y proporcionan hábitat para la fauna silvestre. La vegetación ayuda a la salud mental y física de la población, la provisión de oportunidades de recreación, oportunidades educativas en el tema ambiental y el mejoramiento estético del ambiente (Sorensen, Barzetti, Keipi \& Williams, 1998).

Los parques urbanos poseen una complejidad estructural a partir de sus variables biofísicas, como la vegetación, dimensión, riqueza (De la Herra, Unanue \& Aguirre, 2009), entre más complejo más diverso será. Por tal motivo, el objetivo de este estudio fue caracterizar las variables biofísicas de parques urbanos del Cantón Central de San José. 


\section{MATERIALES Y MÉTODOS}

El trabajo de campo se llevó a cabo entre setiembre y diciembre del 2009, en 15 parques urbanos en la ciudad de San José (capital de Costa Rica) pertenecientes a cuatro distritos del Cantón Central de San José: El Carmen, Catedral, Hospital y Merced (Fig. 1). Los parques urbanos estudiados fueron: Parque Morazán, Jardín de Paz, Parque España, Parque Nacional, Plaza de la Democracia, Parque de las Garantías Sociales, Plaza de las Artes, Plaza González Víquez, Parque Cañas, Parque Dolorosa, Parque Central, Parque Ministerio de Salud, Parque Braulio Carrillo, Bulevar del Correo y Parque Central de Barrio México. La información de la caracterización de las variables biofísicas de los parques se obtuvo mediante la exploración del terreno a partir de un formulario y el análisis de datos suministrados por la sección de parques de la Municipalidad de San José.
Para seleccionar aleatoriamente los parques urbanos bajo estudio, se dividió con líneas imaginarias el Cantón Central de San José en cuatro cuadrantes de 100ha cada uno, dentro de los distritos seleccionados. Se utilizó como punto de referencia calle 0 y avenida 0 definiendo cada cuadrante según los puntos cardinales. Todos los parques y áreas verdes que se encuentren en esa área, fueron tomados como sitios de estudio siempre y cuando presentaran las siguientes variables: 1) área superior $1700 \mathrm{~m}^{2}$, 2) parques urbanos ubicados en un radio de $1 \mathrm{Km}$ a partir de calle 0 avenida 0 y 3 ) administrados por la Municipalidad de San José, ya que este gobierno local está realizando acciones en la temática ambiental. Los parques urbanos en estudio presentan diferentes características entre sí, en cuanto a superficie, coordenadas y cuadrante de ubicación (Cuadro 1).

Caracterización de la infraestructura del parque urbano: Para el registro y la caracterización de las variables

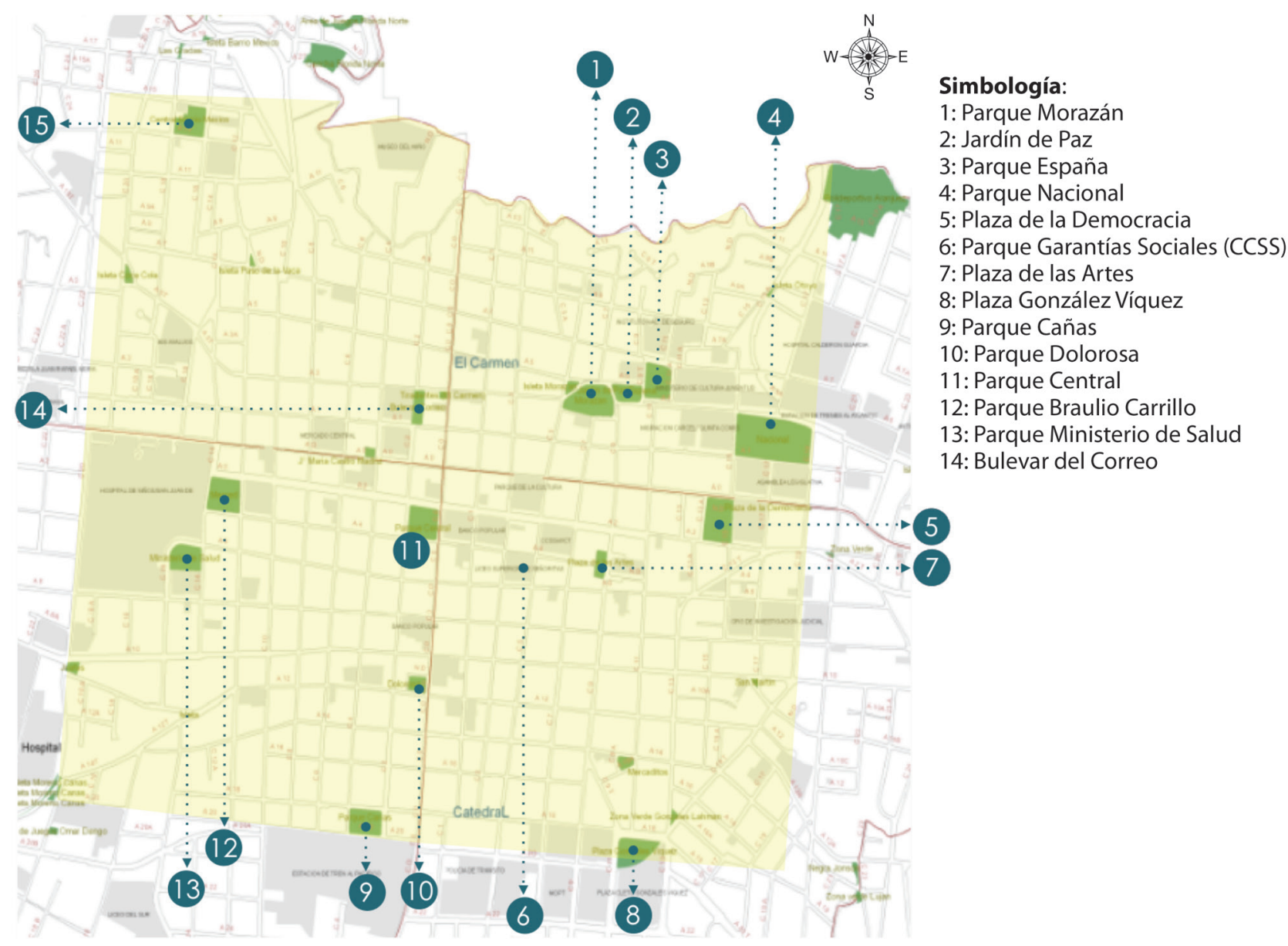

Fig. 1. Distribución de los parques urbanos muestreados del Cantón Central de San José, Costa Rica. Fuente: adaptado de áreas verdes bajo administración de la Sección Parques Municipalidad de San José, 2008 (Fallas, 2016). 
CUADRO 1

Dimensiones y ubicación de los parques urbanos, cantón central de San José costa Rica, 2009.

\begin{tabular}{lccccc}
\multicolumn{1}{c}{ Parque Urbano } & Área $\left(\mathrm{m}^{2}\right)^{*}$ & \multicolumn{2}{c}{ Coordenadas geográficas } & Distrito & Número de Cuadrante \\
& 8626,302 & $9^{\circ} 56^{\prime} 7.98^{\prime \prime}$ & $84^{\circ} 4^{\prime} 31.20^{\prime \prime}$ & Carmen & 1 \\
Parque Morazán & 3104,128 & $9^{\circ} 56^{\prime} 7.95^{\prime \prime}$ & $84^{\circ} 4^{\prime} 27.94^{\prime \prime}$ & Carmen & 1 \\
Jardín de Paz & 5699,297 & $9^{\circ} 56^{\prime} 9.62^{\prime \prime}$ & $84^{\circ} 4^{\prime} 25.43^{\prime \prime}$ & Carmen & 1 \\
Parque España & 21220,944 & $9^{\circ} 56^{\prime} 4.95^{\prime \prime}$ & $84^{\circ} 4^{\prime} 22.49^{\prime \prime}$ & Carmen & 2 \\
Parque Nacional & 8382,026 & $9^{\circ} 55^{\prime} 58.17^{\prime \prime}$ & $84^{\circ} 4^{\prime} 19.80^{\prime \prime}$ & Catedral & 2 \\
Plaza de la Democracia & 5966,770 & $9^{\circ} 55^{\prime} 53.76^{\prime \prime}$ & $84^{\circ} 4^{\prime} 35.13^{\prime \prime}$ & Catedral & 2 \\
Parque Garantías Sociales & 1983,450 & $9^{\circ} 55^{\prime} 54.66^{\prime \prime}$ & $84^{\circ} 4^{\prime} 30.38^{\prime \prime}$ & Catedral \\
Plaza de las Artes & 5992,625 & $9^{\circ} 55^{\prime} 31.37^{\prime \prime}$ & $84^{\circ} 4^{\prime} 27.35^{\prime \prime}$ & Catedral \\
Plaza González Víquez & 5548,491 & $9^{\circ} 55^{\prime} 33.55^{\prime \prime}$ & $84^{\circ} 4^{\prime} 51.68^{\prime \prime}$ & Hospital \\
Parque Cañas & 1786,819 & $9^{\circ} 55^{\prime} 44.90^{\prime \prime}$ & $84^{\circ} 4^{\prime} 47.08^{\prime \prime}$ & Hospital \\
Parque Dolorosa & 6312,072 & $9^{\circ} 55^{\prime} 57.95^{\prime \prime}$ & $84^{\circ} 4^{\prime} 46.05^{\prime \prime}$ & Hospital \\
Parque Central & 6917,514 & $9^{\circ} 56^{\prime} 0.12^{\prime \prime}$ & $84^{\circ} 5^{\prime} 4.54^{\prime \prime}$ & Hospital \\
Parque Braulio Carrillo & 5191,075 & $9^{\circ} 55^{\prime} 55.15^{\prime \prime}$ & $84^{\circ} 5^{\prime} 8.06^{\prime \prime}$ & Hospital & 3 \\
Parque Ministerio de Salud & 2507,196 & $9^{\circ} 56^{\prime} 7.33^{\prime \prime}$ & $84^{\circ} 4^{\prime} 47.09^{\prime \prime}$ & Merced \\
Bulevar del Correo & 5135,817 & $9^{\circ} 56^{\prime} 30.57^{\prime \prime}$ & $84^{\circ} 5^{\prime} 7.57^{\prime \prime}$ & Merced \\
Parque Central de Barrio México & & & 3 \\
\hline
\end{tabular}

*Datos proporcionados por Arias (2009).

biofísicas de los parques urbanos primero se definió perceptualmente el porcentaje de área cubierta por pavimentos y áreas verde (se reafirman los datos con la información facilitada por la Sección de Parques de la Municipalidad de San José). Se determinaron los estratos de vegetación (validados con el material aportado por la sección de parques de la Municipalidad de San José), además, de la identificación de especies vegetales cuando no existiera el levantamiento en de las especies por parte de la sección de parques de la Municipalidad de San José (Fallas, 2016). Las variables biofísicas evaluadas fueron las siguientes: área $\left(\mathrm{m}^{2}\right)$, área zona verde (\%), área obra gris (\%), riqueza de plantas, estratos de vegetación. La riqueza de flora presente en cada parque, fue proporcionada por la Sección de Parques de la Municipalidad de San José y otras se identificaron en el campo. Se identificaron solo árboles y arbustos categorizados según su altura en los siguientes estratos: estrato bajo $(\leq 1,5 \mathrm{~m}$ de altura), estrato medio $(1,5 \mathrm{~m} \leq 5 \mathrm{~m}$ de altura) y estrato alto ( $>5 \mathrm{~m}$ de altura). Las características biofísicas se compararon entre parques y cuadrantes, área $\mathrm{m}^{2}$, riqueza de plantas, estratos de vegetación, infraestructura existente).

\section{RESULTADOS}

Áreas: El área de los parques oscila entre 1786,82 y $21220,94 \mathrm{~m}^{2}$. El cuadrante 1 está conformado por cuatro parques los cuales presentan tamaños entre los 3104,13 - $21220,94 \mathrm{~m}^{2}$, corresponde al cuadrante con mayor cantidad de área en cuanto los parques urbanos. Para los sitios ubicados en el cuadrante 2 (conformado por cuatro parques) se identificaron áreas entre 1983,45 $8382,03 \mathrm{~m}^{2}$, mientras que los sitios que pertenecen al cuadrante número 3 correspondientes a cinco parques, presentan áreas entre $1786,82-6917,51 \mathrm{~m}^{2}$ y el cuarto cuadrante que está conformado únicamente por dos parques, 2507,19 - 5135,82 $\mathrm{m}^{2}$ respectivamente, corresponde al cuadrante con menor cantidad de área en cuanto los parques urbanos.

El cuadrante 1 fue el presentó la mayor área promedio con aproximadamente $9663 \mathrm{~m}^{2}$ de zonas verdes, mientras que el cuadrante 4 es el que presenta menor promedio con aproximadamente $3821 \mathrm{~m}^{2}$. De los parques urbanos, 10 presentaron un área que se encuentra entre los 5000 y $10000 \mathrm{~m}^{2}$; por otro lado, cuatro presentaron un área menor de $5000 \mathrm{~m}^{2}$ siendo el Parque de la Dolorosa en el cuadrante 3 el de área más pequeña $1787,8 \mathrm{~m}^{2}$ aproximadamente y como caso especial, se encuentra el Parque Nacional en el cuadrante 1 con un área que supera los $20000 \mathrm{~m}^{2}$ (Cuadro 1).

Zonas verdes y obra gris: En los parques urbanos se determinaron tres tendencias en cuanto a la relación área de zona verde y área de obra gris: 


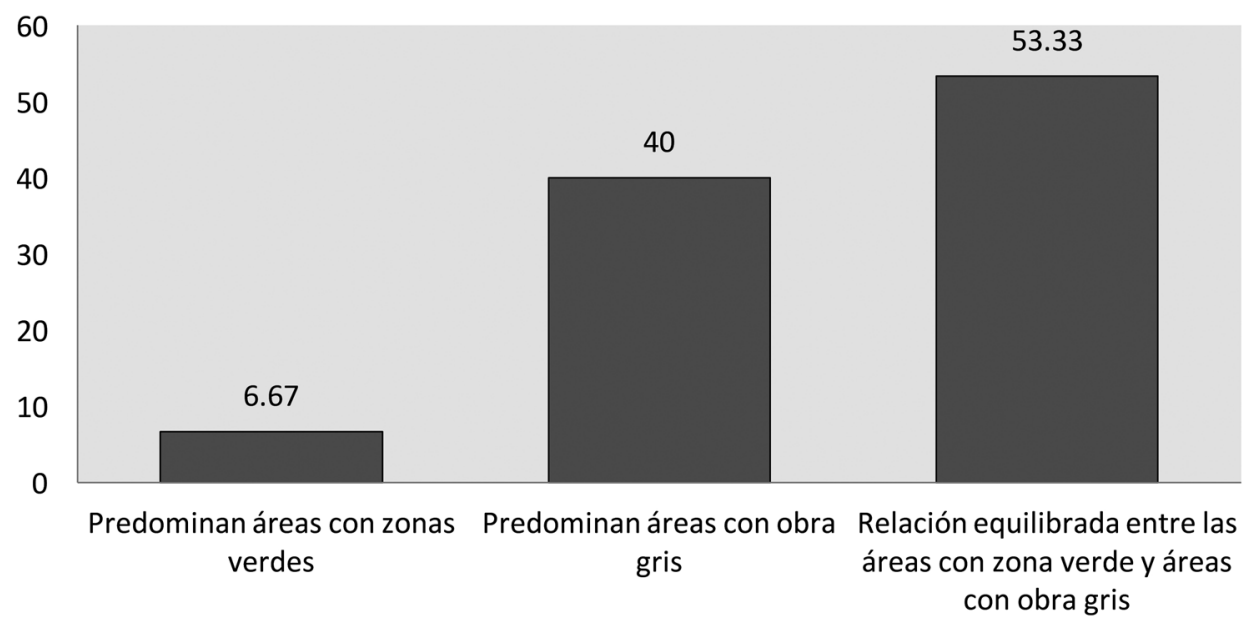

Fig. 2. Porcentaje de área zona verde y área obra gris en los 15 parques urbanos Cantón Central de San José, entre setiembre-diciembre 2009.

a. Predominancia porcentual de áreas con zonas verdes: corresponde a un $6,67 \%$ de los sitios en estudio (Fig. 2). No existen jardineras ya que todo el sitio es una zona enzacatada con sendas que atraviesan el lugar que permiten a los usuarios trasladarse por el sitio.

b. Predominancia porcentual de áreas con obra gris: correspondiente al $40 \%$ del total de parques en estudio (Fig. 2). En estos sitios, se encuentra bien demarcadas las jardineras, es decir que existen zonas que rodean las áreas verdes, creando barreras tanto físicas como visuales, que evitan que el usuario no se desplace por estas y permiten el mantenimiento de las zonas verdes diseñadas; además no existen sendas definidas, ya que la mayoría del área se encuentra en obra gris y el usuario puede desplazarse libremente por casi la totalidad del área. c. Relación proporcional entre las áreas con zona verde y las áreas con obra gris (la diferencia entre una y otra no sobrepasa el $10 \%$ ) correspondiendo al $53,33 \%$ del total de las áreas verdes en estudio (Fig. 2). En estos sitios, existen tanto jardines como sendas bien delimitados, el usuario puede hacer uso tanto de las áreas verdes (como zonas de descanso), como las áreas de obra gris (para desplazarse de un sitio a otro).

Los parques urbanos en estudio, el cuadrante 1 presenta $19531 \mathrm{~m}^{2}$ de áreas verdes y $19872 \mathrm{~m}^{2}$ de áreas con obra gris (Se incluyen cuatro parques urbanos). El cuadrante 2 posee $4237 \mathrm{~m}^{2}$ de áreas verdes y $9705 \mathrm{~m}^{2}$ de obra gris (este dato no es certero ya que no se cuenta con la información referente a la Plaza de la Democracia, incluye únicamente tres de cuatro datos de áreas). El cuadrante 3 presenta $11076 \mathrm{~m}^{2}$ de áreas verdes y $14680 \mathrm{~m}^{2}$ de

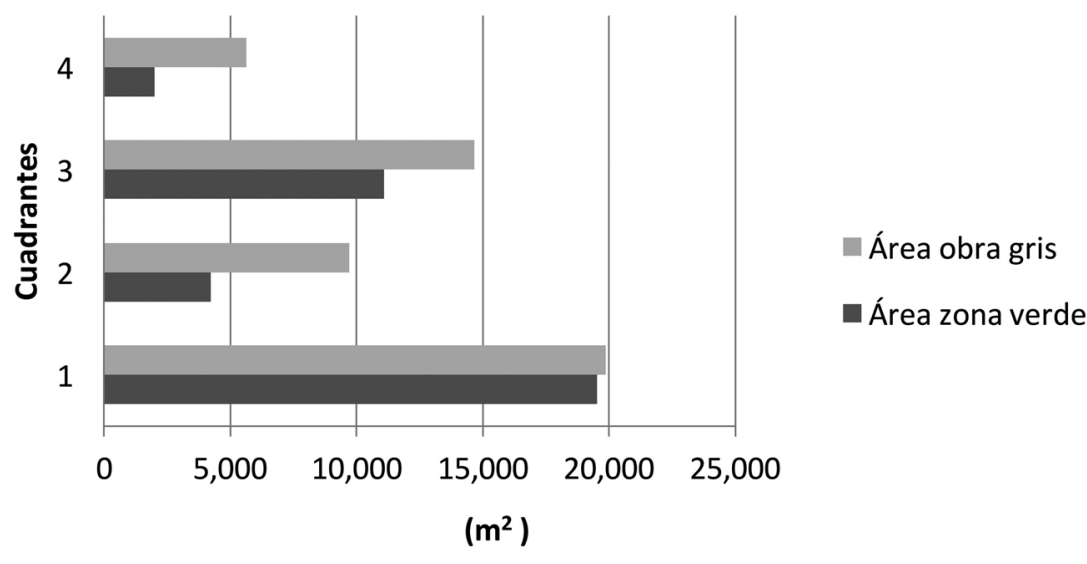

Fig. 3. Áreas $\left(\mathrm{m}^{2}\right)$ con obra gris y áreas con zona verde en los 4 cuadrantes del Cantón Central de San José (Distritos: Carmen, Catedral, Hospital y Merced), entre setiembre-diciembre 2009. 
obra gris (correspondiente a 5 parques urbanos). El cuadrante 4 cuenta con $1993 \mathrm{~m}^{2}$ de áreas verdes, mientras que de áreas con obra gris presenta $5650 \mathrm{~m}^{2}$ de obra gris (correspondiente únicamente a dos parques urbanos). Según las tres tendencias identificadas para comparar áreas con obra verde y áreas con obra gris, el cuadrante 1 presentan una relación proporcional entre las áreas con zona verde y las áreas con obra gris; mientras que el resto de los cuadrantes presentan una tendencia en la cual se da una predominancia porcentual de áreas con obra gris (Fig. 3).

La predominancia porcentual de áreas con zonas verdes se da únicamente en el parque del Ministerio de Salud, la predominancia porcentual de áreas con obra gris está representada por 6 de los sitios donde sobresale el parque Central y en la tendencia donde la relación es proporcional entre las áreas con zona verde y las áreas con obra gris, se incluyen ocho sitios donde sobresale el parque Nacional (Fig. 4).

La caracterización por cuadrante y parque aparece en el Apéndice Digital.

\section{Estratificación general}

Al sobreponer los datos sobre los estratos de cobertura vegetal de los sitios en estudio, se observa una clara dominancia del estrato alto en siete de los 15 sitios donde sobresale el Parque Nacional por la cantidad de individuos con esta condición, mientras que en tres de los sitios sobresale el estrato medio (Fig. 5).

Analizando conjuntamente los estatus de las especies vegetales en los sitios de estudios, se puede mencionar que en la mayoría de los sitios (13 de los 15 sitios) sobresalen las especies exóticas, únicamente en la Plaza de la Democracia las especies vegetales nativas superan a las exóticas (Fig. 6).

Los parques estudiados en el cantón Central de San José, poseen un alto margen de diferencia entre la cantidad de plantas nativas con respecto a las especies exóticas. En general las especies nativas son representadas por un $13 \%$ mientras que las exóticas por el $87 \%$ del total.

\section{DISCUSIÓN}

La importancia de las zonas verdes en las ciudades radica en los efectos positivos que tienen sobre la población residente, efectos que pueden manifestarse en varios ámbitos: i) en el aprendizaje de la conciencia ambiental o ecológica; ii) en el proceso de enraizamiento (embeddedness) en la comunidad y de construcción de identidad cultural y capital social; iii) en el sentimiento de seguridad; y iv) en la mejora del estado de salud mental y física de los ciudadanos (Moyano \& Priego, 2009). Adicional a esto, brindan un hábitat (temporal o permanente) a especies adaptadas a entornos urbanos, en especial a aves tanto residentes como migratorias.

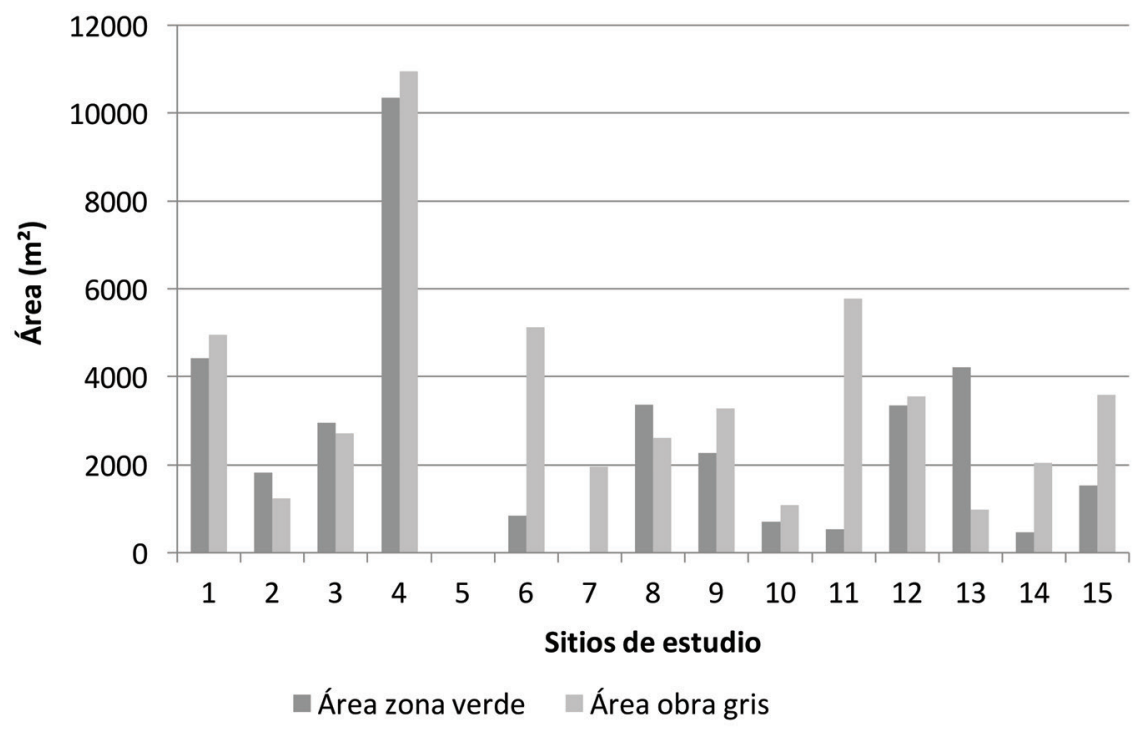

Fig. 4. Áreas con obra gris y áreas con zona verde en los parques urbanos del Cantón Central de San José, entre setiembre-diciembre 2009. 1=Parque Morazán, 2=Jardín de Paz, 3=Parque España, 4=Parque Nacional, 5=Plaza de la Democracia, 6=Parque Garantías Sociales (CCSS), 7=Plaza de las Artes, 8=Plaza González Víquez, 9=Parque Cañas, 10=Parque Dolorosa, 11=Parque Central, 12=Parque Braulio Carrillo, 13=Parque Ministerio de Salud, 14=Bulevar del Correo, 15=Parque Central de Barrio México. 


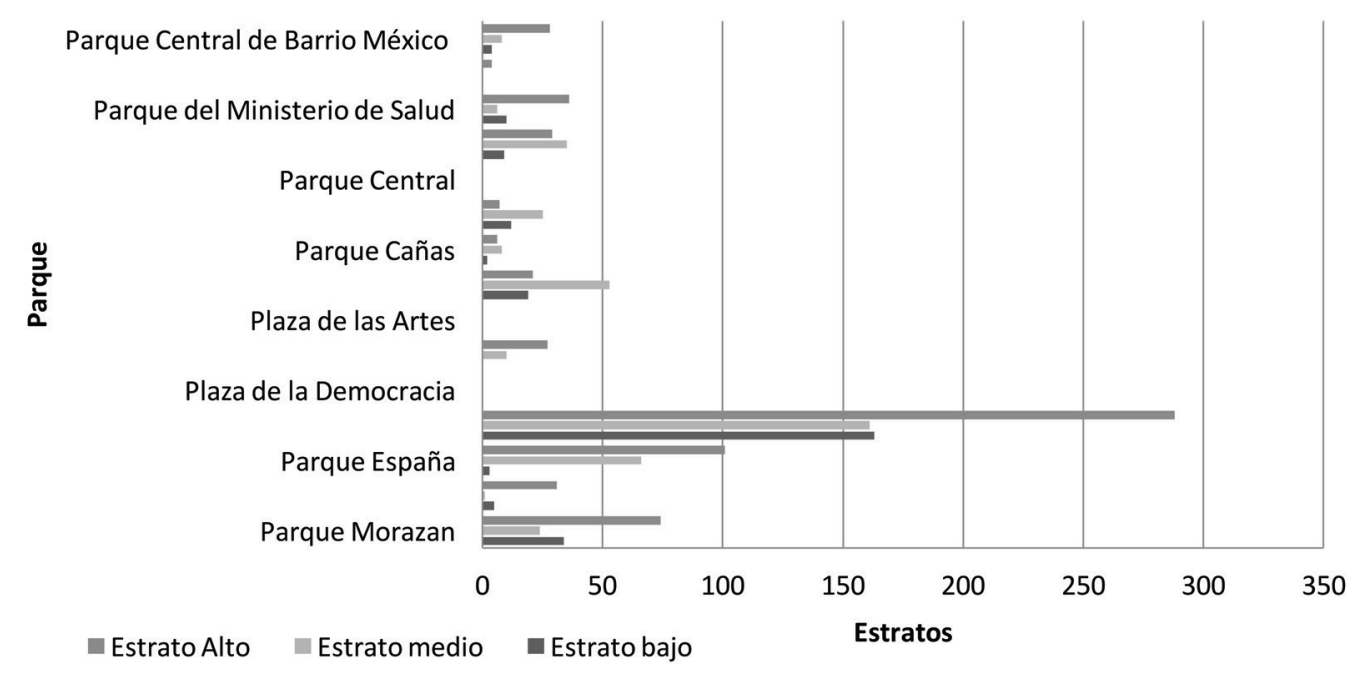

Fig. 5. Estratos de cobertura por sitio de estudio, Cantón Central de San José en el Periodo setiembre-diciembre 2009.

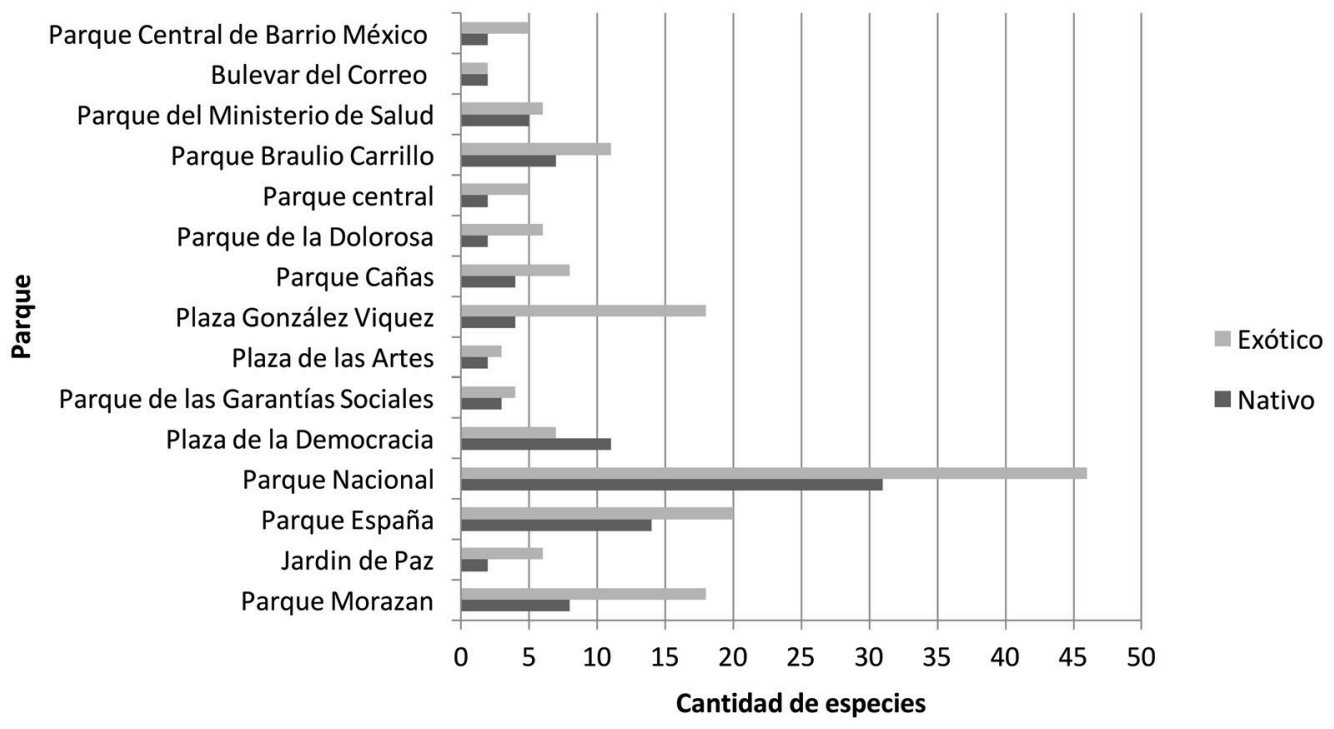

Fig. 6. Cantidad de especies vegetales nativas y exóticas según estatus por sitio de estudio, Cantón Central de San José en el Periodo setiembre-diciembre 2009.

Conejero y Sallent (2011) citando a Sánchez (2011) indican que las áreas verdes urbanas pueden definirse como nódulos de carga, estos corresponden a espacios del territorio que pueden ser utilizados como hábitat temporal o permanente por especies y poblaciones animales proporcionando diferentes tipos de uso (alimentación, reproducción, protección, descanso). Estos nódulos de carga, son de vital importancia dentro del ecosistema urbano, ya que algunos forman parte de una estructura de nudos ecológicamente muy ricos, unidos a una red de conexión territorial con unas características biológicas muy apreciables. Por tal razón, no se puede entender el sistema de nódulos de carga sin una buena conectividad ecológica (Andrés \& Canals, 2011). Para Andrés y Canals (2011) estos nódulos otorgan permeabilidad al sistema urbano $y$, a su vez, soportan los niveles de biodiversidad desde un punto de vista dinámico. Sin embargo, la fragmentación a nivel urbano tiende a ser muy evidente y compromete el aprovechamiento de estos nódulos.

De la Herra et al. (2009), citando a Hinsley et al. (1995) indican que los parques más grandes favorecen el establecimiento de las especies debido a la mayor 
disponibilidad y diversidad de hábitats. Sin embargo, una superficie extensa no es suficiente para mantener una rica diversidad de especies, ya que la riqueza, también responde a la complejidad estructural de los parques (De la Herra et al., 2009). Según Sierra (2012) citando a Vélez (2004) las áreas verdes se prefieren de tamaño pequeño a las grandes, con bordes bien definidos y manejados, lo cual delimitada el potencial de las áreas para albergar gran biodiversidad. Para los sitios en estudio se observa que las áreas de mayor tamaño pueden tener mayor disponibilidad de recursos para la vida silvestre, sin embargo, estas variables no son las únicas que deben contemplarse para determinar la riqueza de especies. La heterogeneidad de estratos y la variación de alturas en la estructura vertical de los bosques pueden favorecer mayor biodiversidad que en el caso de la homogenización de alturas, de árboles maduros y entre ellos solo una cobertura herbácea (Sierra, 2012).

El comportamiento de especies vegetales en los parques estudiados en el cantón Central de San José, se ve superado porcentualmente por especies exóticas, en 14 de los 15 sitios de estudio sobresalen las especies vegetales exóticas, únicamente en la Plaza de la Democracia las especies vegetales nativas superan a las exóticas. Sánchez y Artavia (2013) indican que para la arborización urbana se puede utilizar un margen entre $70-80 \%$ de especies nativas y el resto de especies exóticas. Sin embargo, esta recomendación no se cumple para el cantón Central de San José. Las especies nativas están mejor adaptadas, son tolerantes a cambios de clima, son amigables con diversidad de fauna, son óptimas para la conformación de corredores ambientales y lograr la conectividad de ecosistemas fragmentados por el desarrollo humano" (Barboza, 2016, citando el Manual de Arborización Urbana, 2009). Sin embargo, las especies migratorias de aves ven en las especies exóticas y los estratos altos de vegetación sitios de estancia y alimentación temporal durante su proceso migratorio.

La poca densidad de árboles en un sitio reduce los beneficios que proveen los parques urbanos, como pueden ser: la intervención en procesos de conectividad y funcionalidad de los ecosistemas urbanos, la regulación del microclima, el control de la erosión y el secuestro de carbono (Barboza, 2016, citando a Galeano, 2009), esto es evidente en parques de los cuadrantes 2, 3 y 4 . De igual manera, Barboza (2016) citando a Estrada y Sánchez (2012) indica que mantener cierto grado de cobertura arbórea, incluir un porcentaje de hábitat nativo, son medidas importantes que favorecen la conservación de la diversidad biológica dentro de los paisajes humanizados.

\section{REFERENCIAS}

Andres, O., \& Canals, E. (2011). Estudio del ecosistema urbano de San José: Protocolo de monitoreo de aves y naturalización del Parque La Sabana. Recuperado de http://ddd. uab.cat/pub/trerecpro/2011/hdl_2072_179283/PFC_ EcosistemaUrbaSanJose.pdf

Arias, J. (7 de Octubre de 2009). Parques Urbanos, Municipalidad de San José. (A. A. Solano, Interviewer) San José, Costa Rica.

Barboza, R. (2016). Especies para arbolado urbano. Análisis de 10 parques urbanos del cantón de Curridabat (Tesis de Licenciatura). Universidad Estatal a Distancia. San José, Costa Rica.

Barillas, A. (2005). Estructuras de las comunidades de aves de los parques urbanos de la Ciudad de Puebla México. Recuperado de http://catarina.udlap.mx/u_dl_a/tales/ documentos/laec/de_I_aa/capitulo1.pdf

Buzo, D., \& Hernández, L. (2004). Dinámica espacial y temporal de la comunidad de aves en los parques urbanos de Puebla y su entorno. Puebla, México: Departamento de química y biología, Escuela de Ciencias, Universidad de las Américas Puebla.

Canosa, E., Sáez, E., Sanabria, C., \& Zavala, I. (2003). Metodología para el estudio de Parques Urbanos: la comunidad de Madrid. Revista Internacional de la Ciencia y la Tecnología de la Información Geográfica, 3, 160-185.

Conejero, A., \& Sallent, E. (2011). Estudio del ecosistema urbano de San José, protocolo de monitoreo de aves y naturalización del parque La Sabana. Recuperado de de:http:// ddd.uab.cat/pub/trerecpro/2011/hdl_2072_179283/ PFC_EcosistemaUrbaSanJose_resum.pdf

De la Herra, I., Unanue, A., \& Aguirre, I. (2009). Efectos del área, edad y cobertura de la vegetación sobre la riqueza de especies de aves reproductoras en los parques urbanos de Vitoria-Gasteiz. Munibe Ciencias Naturales, 57, 195-206.

Fallas, A. (2016). Uso de parques urbanos por aves residentes y migratorias en la ciudad de San José, Costa Rica (Tesis de Licenciatura). Universidad Estatal a Distancia. San José, Costa Rica.

Galeano, L. (2009). Evaluación de la inversión pública realizada en las áreas urbanas del corredor ecológico San Lucas-Belén Rincón (Tesis Maestría). Medio ambiente y desarrollo, Universidad Nacional de Colombia. Medellín, Colombia.

Manual de arborización urbana: Guía práctica para la selección, siembra, cuidado y protección de árboles y palmas para zonas blandas y parques de Santiago de Cali. (2009). Santiago de Cali, Colombia

Moyano E., \& Priego, C. (2009). Marco teórico para analizar las relaciones entre paisaje natural, salud y calidad de vida. Sociedad Hoy, 16, 31-44. Universidad de Concepción. Chile. Recuperado de http://digital.csic.es/bitstream/10261/63540/1/Marco\%20Teórico\%20para\%20 
analizar\%20las\%20relaciones\%20entre\%20paisaje\%20 natural,\%20salud\%20y\%20calidad\%20de\%20vida.pdf

Sánchez, G; Artavia, R. (2013). Inventario de la foresta de San José: Gestión ambiental urbana. Ambientico, 232-233, 26-33.

Sección de Parques y áreas verdes, Municipalidad de San José. Parques y áreas verdes, distritos centrales. Levantamiento de campo: SIG-PLANARBU. (2009). San José, CR. Esc. 1:10.000. Color.
Sierra Vásquez, M. (2012). Ciudad y fauna urbana. Un estudio de caso orientado al reconocimiento de la relación hombre, fauna, y hábitat urbano en Medellín. Recuperado de http://www.bdigital.unal.edu.co/6825/1/CIUDAD_Y FAUNA_URBANA._Un_estudio_de_caso_orientado_al_ reconocimiento_de_la_relaci\%C3\%B3n_hombre,fauna_y_h\%C3\%A1bitat_urbano_en_Medell\%C3\%ADn.. pdf

Sorensen, M., Barzetti, V., Keipi, K., \& Williams, J. (1998). Manejo de las áreas verdes urbanas. Documento de buenas prácticas. Washington, D.C, EEUU. 